\title{
Insegurança alimentar e obesidade em adultos: Qual a relação?
}

\author{
Caryna Eurich Mazur ${ }^{1}$, Francisco Navarro²
}

\section{RESUMO}

A obesidade é uma doença multifatorial que tem, entre outros pontos relevantes, a inadequação alimentar. A insegurança alimentar é proposta como sendo o desajuste da alimentação, em qualidade, quantidade e diversidade. Sendo assim, o objetivo desse estudo foi relacionar a obesidade à insegurança alimentar. Esse estudo é uma revisão de literatura que buscou sintetizar a relação entre a insegurança alimentar e a obesidade em adultos. Os trabalhos expostos aqui demonstraram que as mulheres estão mais propensas à desenvolverem obesidade, assim como a insegurança alimentar. Alguns autores consideraram o quesito renda como uma variável de interesse, e obtiveram correlação com a obesidade e a insegurança alimentar. Diante disso, a conclusão foi de que, diante de todos os fatores, há associação entre a insegurança alimentar e a obesidade, de modo que 0 aumento da oferta de alimentos não saudáveis e/ou 0 acesso à esses alimentos pode ampliar 0 aumento do peso corporal, e levar ao quadro de obesidade.

Descritores: Obesidade; Segurança Alimentar e Nutricional; Alimentação; Nutrição.

\section{Food insecurity and obesity in adults: What is the relationship?}

\begin{abstract}
Obesity is a multifactorial disease that has, among other relevant issues, inadequate food. Food insecurity is proposed as the mismatch of supply in quality, quantity and diversity. Therefore, the aim of this study was that obesity and food insecurity are closely linked. Method: This study is a literature review. The works exhibited here have shown that women are more prone to develop obesity, as well as food insecurity. Some felt the question income as a variable of interest, and obtained correlation with obesity and food insecurity. Therefore, the conclusion was that there is an association between food insecurity and obesity, so that the increased supply of unhealthy foods and / or access to these foods can enlarge the increase in body weight, and lead to obesity frame.
\end{abstract}

Descriptors: Obesity; Food Security and Nutrition; Food; Nutrition.

\footnotetext{
${ }^{1}$ Nutricionista, especialista em Obesidade e Emagrecimento pela Universidade Gama Filho (UGF), Rio de Janeiro, RJ, Brasil.

${ }^{2}$ Doutor em Ciências pela Universidade de São Paulo (USP), São Paulo, SP, Brasil.
} 


\section{Introdução}

A alimentação é fundamental para o bem-estar, desenvolvimento humano e para alcançar a segurança alimentar e nutricional. No entanto, apesar da produção mundial de alimentos ao longo do último século manter-se à frente da demanda global, hoje cerca de um bilhão de pessoas não têm o suficiente para comer, e uma nutrição adequada falta em mais de um bilhão adicionais a isso ${ }^{1,2}$.

O estado nutricional é o resultado da ingestão alimentar e da necessidade nutricional e do estado de saúde, que está relacionado com fatores causais, principalmente aqueles à nível familiar, ambiental, demográfico entre outros. As causas subjacentes da ingestão de alimentos e o estado de saúde podem ser influenciadas por diversos determinantes, entre eles 0 aspecto social e econômico. Há diferenças entre segurança alimentar e segurança nutricional. A segurança alimentar refere-se à disponibilidade de alimentos a nível doméstico (por exemplo, o acesso ao alimento) e utilização (por exemplo, a higiene alimentar). As causas subjacentes do estado de saúde (causas ambientais e serviços de saúde) podem ter vários fatores que contribuem (por exemplo, habitação e acesso limitado à assistência a saúde) para a IA. A segurança nutricional engloba toda essa relação entre o ambiente, sociedade e alimentação. Sendo assim, a segurança nutricional existe quando é alcançado 0 adequado estado nutricional ${ }^{3}$.

Nos últimos anos o mercado ofereceu comida barata, saborosa e rica em gordura, o mais energético dos macronutrientes. Sabe-se que o consumo involuntário de calorias é facilmente estimulado por alimentos ricos em gordura ${ }^{4}$. Nesse sentido, a combinação de fatores econômicos e biológicos, provavelmente, resulta em um acúmulo de calorias que advém desses alimentos baratos e prontamente disponíveis. $O$ consumo de alimentos reflete na interação entre duas partes do cérebro: um sistema "deliberativo" que toma decisões e um sistema "afetivo" que responde rapidamente a estímulos. O quadro de dupla decisão é caracterizado por comer demais e pelo excesso de peso $0^{5}$.

A obesidade é uma doença crônica, além de ser um dos principais problemas de saúde pública no mundo, está rapidamente tornando-se uma epidemia que contribui na carga global de doenças correlatas na população. A obesidade, especialmente a central, é importante fator de risco para diabetes tipo 2, hipertensão, doenças cardiovasculares além de alguns tipos de câncer ${ }^{6}$.

Nos países desenvolvidos, a condição socioeconômica é inversamente proporcional à obesidade. Existe, também, a tendência clara do decréscimo da prevalência de obesidade à medida que aumenta o nível de escolaridade. Apesar da prevalência da obesidade diferir de acordo com a condição socioeconômica, os aumentos ao longo do tempo parecem ser similares em todos os grupos socioeconômicos ${ }^{7}$.

Sob outra ótica, o termo ambiente obesogênico resume a participação de componentes econômicos, sociais, culturais e psíquicos na oferta de alimentos, no padrão de consumo, na eleição ou na imposição dos horários das refeições e no comportamento sedentário, que contribuem para o gasto calórico diário4.

$\mathrm{O}$ aumento da prevalência de obesidade e sua possível associação com a insegurança alimentar (IA) entre famílias de baixa renda têm levantado se a relação entre segurança alimentar e excesso de peso é uma causa diretå .

Dietz ${ }^{9}$ foi o primeiro a sugerir que a IA pode ser um dos colaboradores subjacentes à obesidade, e atribuiu essa associação para a possível inclusão de mais alimentos com alto teor de gordura e densidade energética, o que diminui a qualidade nutricional da dieta nos indivíduos em situação de IA.

Nesse sentido, o objetivo do presente trabalho foi analisar, segundo a literatura cientifica, a relação da IA com a obesidade em adultos.

\section{Metodologia}

Realizou-se ampla revisão procurando capturar artigos científicos que descrevessem a associação entre IA e a presença de obesidade em adultos.

Esse estudo foi do tipo revisão de literatura, dos últimos dez anos (publicados entre os anos 2002 e 2012). Foram realizadas buscas dos trabalhos nas bases de dados: MEDLINE (Pubmed), LILACS, Cocharne e Scielo, em português e inglês, por meio dos seguintes descritores: obesity and food insecurity e obesidade e insegurança alimentar. As buscas dos artigos originais foram efetivadas no mês de outubro de 2012. Foram usados também livros-textos recentes, considerando a relevância e o valor informativo do material e alguns artigos-chave selecionados a partir de citações em outros artigos.

Como critérios de inclusão foram estabelecidos que apenas pesquisas realizadas em seres humanos, adultos, publicadas nos últimos 10 anos, nos idiomas português, espanhol e inglês seriam selecionadas. Não foram selecionados trabalhos com abordagem qualitativa e com crianças e adolescentes. Os resultados foram expressos de forma descritiva. 


\section{Resultados e Discussão}

Segurança alimentar e nutricional: uma breve conceituação

Os processos de transição demográfica, epidemiológica e nutricional que têm acontecido nas últimas décadas pelo mundo manifestaram-se também no Brasil e têm relação direta com o perfil de consumo alimentar da população e sua morbimortalidade. Novas demandas geradas pelo modo de vida urbano, junto com outras tendências da população, impõem uma adequação dos estilos de vida, em particular dos hábitos alimentares, às novas condições de tempo, recursos financeiros e trabalho. Aliás, a alta exposição à propaganda e publicidade e os produtos desenvolvidos pela indústria e comércio para atender a esta demanda passam a influenciar cada vez mais as escolhas alimentares. É essencial que as políticas públicas de segurança alimentar e nutricional (SAN) efetivamente vinculem a discussão do acesso ao alimento com a adequação da alimentação, o que envolve todo o sistema alimentar, desde as formas de produção até a compra de alimentos, facilitando e estimulando escolhas alimentares saudáveis ${ }^{10}$.

ASAN pode ser definida como sendo o direito humano ao acesso regular e permanente a alimentos de qualidade, em quantidade suficiente, sem comprometimento ao acesso a outras necessidades essenciais, tendo como base práticas alimentares promotoras de saúde, que respeitem a heterogeneidade cultural e que sejam social, econômica e ambientalmente sustentáveis ${ }^{11,12}$.

Uma alimentação que siga estes princípios é capaz de prevenir e reverter doenças carenciais (desnutrição, anemia entre outros) e doenças crônicas degenerativas (obesidade, hipertensão, doenças cardiovasculares). Ao mesmo tempo, seria capaz de estimular o fortalecimento de sistemas sustentáveis de produção de alimentos, no conjunto de uma proposta de desenvolvimento humano sustentável ${ }^{13}$.

Ao longo dos anos 1970 e 1980 a SAN estava predominantemente focada no acesso aos alimentos básicos, como cereais e tubérculos, para resolver problemas de desnutrição energético-proteica. Isso começou a mudar no final dos anos 1980, quando as conclusões de pesquisas em saúde e nutrição, (1) começaram a demonstrar o papel da utilização eficaz dos alimentos na prevenção de doenças, e, (2) destacaram a importância das deficiências dos micronutrientes na saúde. Hoje, apesar de um bilhão de pessoas estarem subnutridas com ingestão insuficiente de proteína e energia, há também o excesso de alimentação - sobrepeso e obesidade. De fato, o paradoxo do fenômeno da obesidade e o que ela está se tornando não é mais apenas um atributo dos ricos que podem pagar para entrar em excesso de consumo, mas mais um fardo dos pobres em todo o mundo ${ }^{14}$.

Diante de uma alimentação inadequada a principal consequência é a IA e a obesidade que constituem uma dimensão de pobreza e de desigualdade social. O consumo alimentar é um indicador importante para a caracterização da pobreza. É relativo presumir que o aumento do rendimento médio dos países mais pobres tenha impacto nos níveis de SAN ${ }^{15}$.

Existem inúmeros fatores pelos quais a obesidade pode estar associada à IA, dentre eles estão a falta de acesso à alimentos saudáveis, as escolhas alimentares erradas, a grande quantidade de alimentos ingeridos, a não diversificação da alimentação com consequente baixa qualidade nutricional da dieta (figura I).

Figura I - Determinantes da relação entre obesidade e insegurança alimentar e nutricional

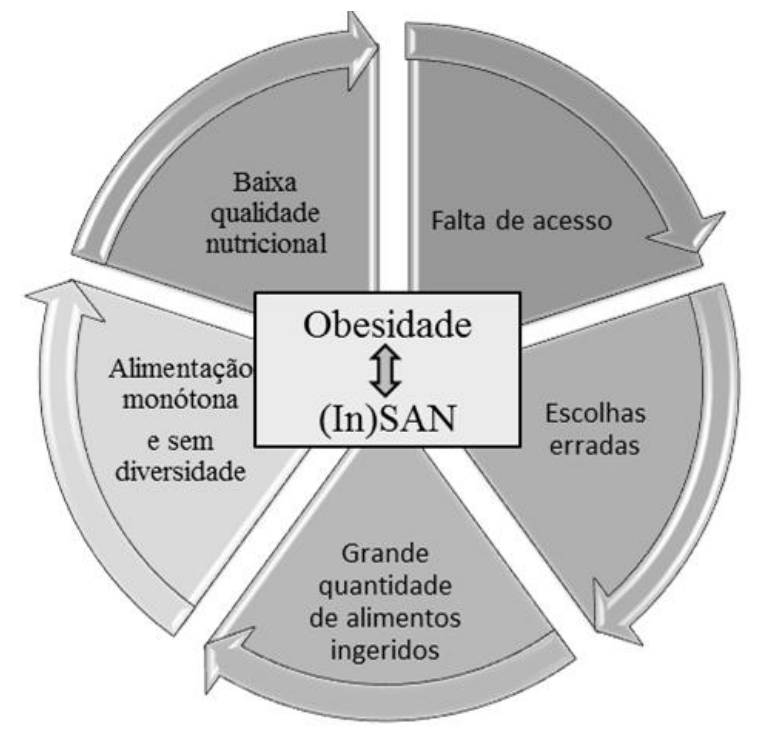


Um dos grandes desafios da atualidade é a busca por estratégias de enfrentamento da IA que não levem ao excesso de consumo e os problemas de excesso de peso. Isto pode ser de interesse para países em desenvolvimento, como muitos na América Latina, que já mostram elevada prevalência de obesidade ${ }^{16}$.

A inclusão da obesidade no contexto da SAN agregou valor à dimensão qualitativa, em seu próprio conceito. Neste enfoque, além das dimensões de dignidade humana, quantidade, diversidade e sustentabilidade, a qualidade da alimentação torna-se também um objetivo a ser adquirido. Enfim, a alimentação saudável e adequada, incorpora-se definitivamente à busca pela garantia da SAN ${ }^{17}$.

Ainda assim, o reforço da medição e investigação continuada da SAN está garantido. Porém, ferramentas atuais não distinguem IA aguda e crônica. Além disso, poucas pesquisas longitudinais avaliam a IA e obesidade em vários pontos temporais ${ }^{18}$.

\section{Obesidade: A grande epidemia}

Pela primeira vez na história do planeta o número de pessoas com excesso de peso supera 0 de desnutridos ${ }^{19}$. Os números mundiais da prevalência de obesidade quase dobraram desde 1980. Em 2008, mais de 1,4 bilhão de adultos estavam acima do peso. Destes, mais de 200 milhões de homens e quase 300 milhões de mulheres eram obesos ${ }^{20}$.

No Brasil esse fenômeno é marcante, com o aumento da prevalência em adolescentes, adultos e idosos. Os estudos sobre os fatores da dieta associados à obesidade concretizados nas últimas décadas no século XX priorizam isolar elementos específicos da dieta, como, por exemplo, as gorduras, as fibras ou o tipo de carboidrato. Essas estratégias não se mostram eficazes para a prevenção da obesidade, e as decisões mais recentes que são adotadas pelos órgãos internacionais e agências de saúde, é abordar, simultaneamente, tanto o consumo quanto o gasto de energia ${ }^{21}$.

A obesidade é uma condição determinada pelo excesso de gordura corporal. No entanto, devido à dificuldade em se obter medidas precisas da gordura corporal na população, as medidas de altura e peso corporal têm sido utilizadas para identificar o sobrepeso e a obesidade. Atualmente, define-se obesidade a partir do índice de massa corporal (IMC) ${ }^{7}$. A IA pode ser considerada um preditor para a obesidade. Mas o oposto também pode aplicar-se. A obesidade pode ser medida de forma objetiva e é de fácil diagnóstico ${ }^{22}$.

Segundo Campos et al. ${ }^{23}$, reporta-se a muitos autores que a discussão sobre a obesidade é muito mais ampla que simples "epidemia mundial". Haveria, por um lado, o interesse dos que lucram com a indústria da obesidade - remédios, dietas, equipamentos - e, por outro lado, os que ficam segregados por sua corpulência.

Sabe-se que a obesidade é uma doença crônica associada à elevada morbimortalidade. As comorbidades maiores para um paciente obeso são: hipertensão arterial, doença cardiovascular, dislipidemia, diabetes tipo 2, síndrome da hipoventilação, apneia do sono, osteoartrite, infertilidade, além dos fatores ambientais (figura II) ${ }^{24}$.

Figura II - Fatores ambientais que exercem pressão constante em direção ao desequilíbrio energético positivo e ao aumento da massa de gordura corporal.

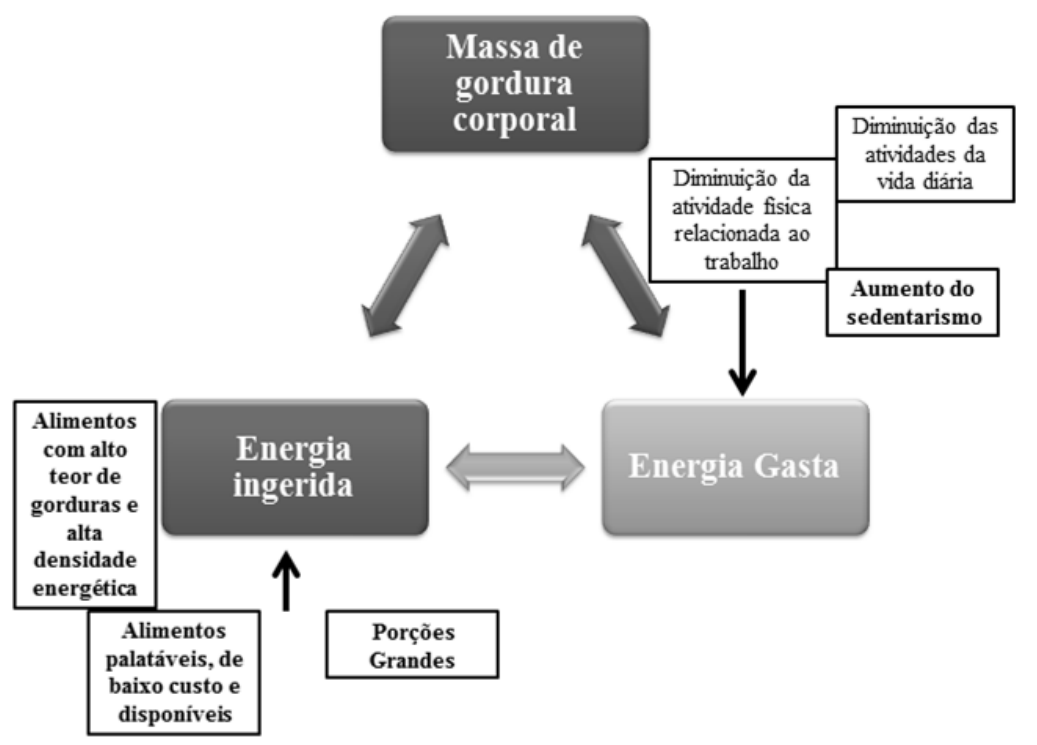

Adaptado de Shils et al. ${ }^{7}$ 
Globalmente, o processo de urbanização foi acompanhado por mudanças de comportamento, principalmente com relação à dieta e à atividade física, privilegiando uma alimentação industrializada com maior oferta de energia, rica em carboidratos simples e gorduras, ao mesmo tempo em que o estilo de vida pode levar ao sedentarismo ${ }^{25}$.

No Brasil em 2008/2009, foi constatado que 1,55 milhões de adultos apresentavam obesidade mórbida, totalizando $0,81 \%$ da população do país, com maior prevalência na Região Sul, nas mulheres e em pessoas de cor preta ${ }^{26}$. Em 2011 , os custos atribuíveis à obesidade totalizaram $\mathrm{R} \$ 487,98$ milhões representando $1,9 \%$ dos gastos com assistência à saúde de média e alta complexidade. Os custos da obesidade mórbida perfizeram $23,8 \%$ dos custos da obesidade (R\$ 116,2 milhões), apesar de sua prevalência ser 18 vezes menor. Os custos com a cirurgia bariátrica no Brasil foram de $R \$$ 31,5 milhões. A obesidade mórbida tem seu custo proporcionalmente 4,3 vezes maior do que 0 da obesidade ${ }^{27}$.

O paradoxo entre fome e obesidade parece, primariamente, inexplicável. No entanto, tal associação é o resultado da IA que procede a inadequação de recursos econômicos para a compra de alimentos, o que constitui a obesidade como uma consequência do consumo alimentar excessivo de escolhas alimentares não saudáveis. A limitação de recursos financeiros levaria à compra de alimentos mais baratos, normalmente mais densidade calórica, à redução do consumo de vegetais e frutas, além de que quando a preocupação com a alimentação gerada por uma situação de IA poderia levar a situações de estresse e depressão, levando a redução da realização de atividade física e constituindo risco para 0 aparecimento da obesidade ${ }^{28}$.

Os fatores que influenciam na ingestão de alimentos estão profundamente enraizados na cultura de cada povo, incluindo fatores econômicos e psicológicos associados com a experiência de longo tempo da pobreza. Sem intervenção, o ciclo da pobreza e da obesidade, de geração em geração, é provável que continue. Estudos têm demonstrado que a pobreza na infância, embora não sempre associada com a obesidade infantil, está associada com níveis mais elevados de obesidade em adultos. Embora o tratamento das desigualdades sociais é difícil para a sociedade, a quebra desse ciclo pode melhorar os efeitos da pobreza, incluindo a obesidade e a IA, com a inserção de alimentos saudáveis e incentivo à atividade física ${ }^{29}$.

As metas atuais de saúde pública em torno da prevenção da obesidade começam a refletir para uma compreensão da potência de políticas públicas e mudanças ambientais para influenciar tanto mudança comportamental e individual ao nível da população, enquanto que o reconhecimento da importância da promoção da SAN é parte de tais esforços. A implementação de políticas públicas em um nível macro, incluindo local, estadual ou federal, é um meio eficaz e abrangente para afetar a mudança no comportamento da população. Alterações no nível de política agem nos ambientes que posteriormente irão influenciar o comportamento e as escolhas dos indivíduos. As políticas eficazes podem mudar ambientes para o melhor acesso (e custos mais baixos) dos alimentos mais saudáveis e, após isso, realizar a escolha saudável (quadro I) ${ }^{30}$.

Quadro I - Incentivos para mudanças nos ambientes macro e micro para influenciar obesidade em populações em IA.

\begin{tabular}{|c|c|c|c|c|}
\hline \multicolumn{5}{|c|}{ Tipo de ambiente } \\
\hline & Físico & Econômico & Político & Sociocultural \\
\hline $\begin{array}{c}\text { Definições Macro } \\
\text { ambientais }\end{array}$ & $\begin{array}{l}\text { Modificações } \\
\text { agrícolas, } \\
\text { habitação, } \\
\text { transporte, } \\
\text { e políticas sociais } \\
\text { que influenciam a } \\
\text { produção e } \\
\text { distribuição de } \\
\text { alimentos. }\end{array}$ & $\begin{array}{l}\text { Oferecer incentivos } \\
\text { monetários } \\
\text { para opções } \\
\text { alimentares } \\
\text { saudáveis e } \\
\text { desincentivos - } \\
\text { impostos - para } \\
\text { opções não } \\
\text { saudáveis. }\end{array}$ & $\begin{array}{c}\text { Promover a } \\
\text { agricultura, } \\
\text { segurança social, } \\
\text { e políticas públicas } \\
\text { de nutrição e } \\
\text { alimentos. }\end{array}$ & $\begin{array}{l}\text { Comercialização de } \\
\text { alimentos e } \\
\text { publicidade correta; } \\
\text { zonas } \\
\text { desfavorecidas } \\
\text { para promoção da } \\
\text { saúde e prevenção } \\
\text { da obesidade. }\end{array}$ \\
\hline $\begin{array}{c}\text { Definições Micro } \\
\text { ambientais }\end{array}$ & $\begin{array}{l}\text { Varejistas de } \\
\text { alimentos e } \\
\text { Pontos de } \\
\text { atendimento } \\
\text { determinam os } \\
\text { locais e opções de } \\
\text { alimentos } \\
\text { saudáveis. }\end{array}$ & $\begin{array}{l}\text { Apoio à saúde } \\
\text { (promoção) à } \\
\text { programas de } \\
\text { nutrição suporte } \\
\text { financeiro para } \\
\text { apoio da } \\
\text { Produção local de } \\
\text { alimentos. }\end{array}$ & $\begin{array}{c}\text { Regras } \\
\text { institucionais e } \\
\text { políticas que } \\
\text { influenciam } \\
\text { disponibilidade } \\
\text { de opções } \\
\text { de alimentos } \\
\text { saudáveis. }\end{array}$ & $\begin{array}{c}\text { Institucionalização } \\
\text { em torno de uma } \\
\text { alimentação } \\
\text { variada } \\
\text { e manutenção } \\
\text { de peso corporal } \\
\text { saudável. }\end{array}$ \\
\hline
\end{tabular}

Adaptado de Rutten et al. ${ }^{30}$. 


\section{Resultados}

Foram encontrados 896 artigos, dos quais, após análise dos critérios de inclusão, 32 artigos fizeram parte desta revisão.

A relação dos artigos selecionados, métodos de investigação, as características gerais da amostra e objetivo do estudo estão descritos sumariamente na tabela 1. Os artigos que constam na tabela foram selecionados conforme seu impacto científico, número populacional e validade.

Em estudo realizado com mulheres, para evidenciar se a IA estava associada ao risco de obesidade, foi confirmado que aquelas que estavam em IA tinham a possibilidade de ter $47 \%$ mais obesidade, ou 2,8 vezes mais chances de estarem com sobrepeso ${ }^{32}$. Em outro estudo com mulheres latinas, demonstrou-se que houve associação fraca e negativa entre a obesidade e a IA $(r=-0,20 ; p<0,001)^{31}$. Resultados semelhantes foram encontrados no estudo de Jilcott et al. ${ }^{33}$.

Estudo de Shariff e $\mathrm{Khor}^{34}$ demonstrou que quando houve a separação da amostra em grupos conforme 0 estado nutricional de mulheres (eutrofia, sobrepeso e obesidade) obteve-se diferença significativa $(p<0,01)$ quando comparado com a existência de IA, de modo que quanto mais obesa, maior a IA em que ela e sua família viviam. Nesse mesmo estudo, 0 acesso aos alimentos também teve associação com a IA, demonstrando que quanto mais alimentos tem-se acesso SAN a pessoa encontra-se em eutrofia $(p<0,01)$. Resultado semelhante foi encontrado no estudo de Wilde e Peterman ${ }^{35}$.

Em outro estudo, buscou-se a relação das famílias com sobrepeso/obesidade e a IA. Foi demonstrado que os pais que eram obesos tinham 2,4 (IC95\%=1,02-8,39) vezes mais chance de terem IA em seus lares, porém sem significância estatística ${ }^{36}$.

Quando é comparado o estado civil e a obesidade dos adultos que vivem em IA o que se tem é que mulheres que são "chefes de família" vivem em maior desconforto com relação à alimentação e à obesidade ${ }^{37}$.

A associação entre a IA e diabetes foi evidenciada, de modo que o diabético tem 2,2 (IC95\%: 1,2-3,9) vezes mais chance de sofrer de $\mathrm{IA}^{38}$. Outra comorbidade comum na obesidade, a dislipidemia, também provou estar relacionada à IA mais em mulheres que em homens. Os valores altos de triglicerídeos tinham 1,9 (IC95\%=1,00-3,63) vezes mais chance de serem encontrados em mulheres com IA e nutricional, porém sem significância estatística; enquanto que o LDL-C alterado tinha 1,85 (IC95\%=1,06-3,23) vezes mais chance de ser encontrado na mesma população, também sem significância estatística ${ }^{39}$.

Em estudo com mulheres grávidas do meio rural, foi demonstrado que a IA no início da gravidez não era associada com aumento do risco de obesidade em dois anos após o parto. A obesidade na gravidez na adolescência foi significativamente associada com o risco aumentado de IA em dois anos após o parto ${ }^{40}$.

Em estudo de Oliveira et al. ${ }^{41}$, no nordeste brasileiro, foi demonstrado que entre adultos e adolescentes a IA estava presente conforme havia o percentual maior desses com sobrepeso/obesidade, porém sem diferenças estatísticas.

Outro estudo brasileiro comprovou que famílias em condições socioeconômicas menos favorecidas apresentaram maior prevalência de IA. A aglomeração no domicílio é uma variável que tem sido relacionada à $I A^{42}$. Portanto, quanto menor poder aquisitivo maior a IA, o que pode gerar também o quadro de sobrepeso.

Em pesquisa realizada com imigrantes fazendeiros que viviam nos Estados Unidos, foi demonstrado que os que estavam em IA em geral, apresentavam maior idade, gastavam 30\% de sua renda com comida, e tinham sobrepeso ${ }^{43}$.

Velásquez-Melendez et al. ${ }^{44}$ explicaram que quando comparadas as mulheres obesas, entre os diferentes níveis de IA (alcançados pela Escala Brasileira de Insegurança Alimentar), a IA moderada e leve apresentaram mais associação com a obesidade (OR=1,53; IC95\%=1,22-1,93 e OR=1,22; IC95\%=1,04-1,44, respectivamente).

Em outro estudo foi demonstrado que em adultos obesos e com IA há mais chance desses terem mais anos de estudo $(\mathrm{OR}=1,83$; IC95\%=1,44-2,33); e quando a regressão logística foi ajustada para idade, sexo, etnia, escolaridade, renda familiar, estado civil, emprego, e número de crianças no domicílio, observou-se $1,3($ IC95\%=1,17=1,50) vezes mais chance de o chefe da família ser obeso e a família viver em IA e nutricional ${ }^{45}$.

É imporante ressaltar que em famílias que vivem em domicílios com IA leve a grave há maior consumo de dietas monótonas, em que a quantidade de comida é insuficiente e compostas principalmente de alimentos como cereais, óleos, açúcar e o consumo de frutas e verduras é baixo ${ }^{46}$. Além disso, 0 custo dos alimentos concebe quase dois terços da renda total em domicílios com IA ${ }^{47}$.

Possíveis teorias propostas para explicar a associação entre sobrepeso e obesidade com a IA incluem: (1) aumento no consumo de alimentos de baixo custo com alta densidade calórica; (2) transtornos alimentares decorrentes da ansiedade e incerteza associadas à restrição ou aumento do consumo alimentar involuntário; (3) adaptações metabólicas a períodos de jejum constantes, até mesmo no período intrauterino, em situação de desnutrição das gestantes ${ }^{48,49,50}$. Em vez de conceituar o problema de sobrepeso como sendo um problema oposto à fome, deve-se considerar a possibilidade de que seja, em populações de baixos rendimentos, uma consequência dela ${ }^{51}$. 
Jones e Frongill ${ }^{52}$ sugerem que a análise dos padrões de ganho de peso entre as mulheres que estão acima do peso inicialmente e a experiência com a IA pode ser uma área fértil para pesquisa longitudinal, que pode ser realizada em diferentes faixas etárias, incluindo em crianças.

Tabela 1 - Relação de artigos selecionados sobre métodos de investigação, conforme autoria

e ano de publicação, características gerais da amostra e objetivo do estudo (2003 a 2012)

\begin{tabular}{|c|c|c|c|c|}
\hline Autor(es) & Ano & Objetivo & $\begin{array}{l}\text { Característica da } \\
\text { amostra }\end{array}$ & Método de investigação \\
\hline Adams et al. ${ }^{32}$ & 2003 & $\begin{array}{l}\text { Determinar a prevalência } \\
\text { de IA com e sem fome em } \\
\text { mulheres da Califórnia e } \\
\text { examinar a relação de IA } \\
\text { com obesidade }\end{array}$ & $\begin{array}{l}\text { Mulheres californianas } \\
\text { adultas }(n=8169)\end{array}$ & $\begin{array}{l}\text { Questionário próprio relacionando fome e IA; } \\
\text { Peso e altura auto relatados. }\end{array}$ \\
\hline Kaiser et al. ${ }^{31}$ & 2004 & $\begin{array}{c}\text { Examinar a relação entre IA } \\
\text { e sobrepeso/obesidade em } \\
\text { mulheres latinas }\end{array}$ & $\begin{array}{l}\text { Mulheres latinas } \\
\text { adultas que viviam no } \\
\text { estado da Califórnia } \\
\text { - Estados Unidos } \\
\quad(n=559)\end{array}$ & $\begin{array}{l}\text { Questionário não validado relacionando fome } \\
\text { e IA; Perguntaram se a mulher considerava- } \\
\text { se obesa. }\end{array}$ \\
\hline $\begin{array}{l}\text { Shariff e } \\
\text { Khor }^{34}\end{array}$ & 2005 & $\begin{array}{l}\text { Determinar os fatores } \\
\text { relacionados à } \mathrm{IAe} \\
\text { sobrepeso em mulheres } \\
\text { ruralistas }\end{array}$ & $\begin{array}{l}\text { Mulheres ruralistas } \\
\text { adultas do estado de } \\
\text { Selangor na Malásia } \\
\qquad(n=200)\end{array}$ & $\begin{array}{l}\text { Questionário demográfico, socioeconômico, } \\
\text { recordatório de } 24 \text { horas e acesso aos } \\
\text { alimentos } \\
\text { Aferidas medidas de peso e altura }\end{array}$ \\
\hline $\begin{array}{l}\text { Wilde e } \\
\text { Peterman }{ }^{35}\end{array}$ & 2006 & $\begin{array}{l}\text { Verificar a influência da IA } \\
\text { no estresse familiar, com } \\
\text { consequente ganho de peso } \\
\text { na mulher }\end{array}$ & $\begin{array}{l}\text { Mulheres americanas } \\
\text { adultas }(n=5303)\end{array}$ & $\begin{array}{l}\text { Questionário validado por Nord et al. } .^{52} \\
\text { contendo } 18 \text { itens relacionando com IA; } \\
\text { avaliando condições socioeconômicas e } \\
\text { também antropométricas (relatados) }\end{array}$ \\
\hline $\begin{array}{l}\text { Hanson, Sobal } \\
\text { e Frongillo }\end{array}$ & 2007 & $\begin{array}{l}\text { Associar IA, peso corporal } \\
\text { entre mulheres e homens. }\end{array}$ & $\begin{array}{l}\text { Homens americanos } \\
(n=4338) \text { e mulheres } \\
\text { americanas }(n=4172) \text {. }\end{array}$ & $\begin{array}{l}\text { Medidas antropométricas (relatadas), } \\
\text { estado civil, variáveis socioeconômicas, e o } \\
\text { questionário Food Security Survey Module. }\end{array}$ \\
\hline $\begin{array}{l}\text { Lyons, Park e } \\
\text { Nelson }{ }^{54}\end{array}$ & 2008 & $\begin{array}{c}\text { Associar obesidade e } \\
\text { segurança alimentar, com } \\
\text { avaliação de dados sobre a } \\
\text { altura e peso aferidos e auto } \\
\text { relatados }\end{array}$ & $\begin{array}{l}\text { Adolescentes, adultos } \\
\text { e idosos canadenses } \\
\qquad(n=35107)\end{array}$ & $\begin{array}{c}\text { Medidas antropométricas (relatadas e, em } \\
\text { alguns, aferida), variáveis sociodemográficas, } \\
\text { SAN medida por meio de } 3 \text { questionários: } \\
\text { Dimensional model of food insecurity, Levels } \\
\text { model of food insecurity e Overall food } \\
\text { insecurity model. }\end{array}$ \\
\hline Oliveira et al. ${ }^{49}$ & 2009 & $\begin{array}{l}\text { Descrever a situação da } \\
\text { (in)segurança alimentar e } \\
\text { sua relação com o estado } \\
\text { nutricional de adolescentes } \\
\text { e adultos }\end{array}$ & $\begin{array}{c}\text { Adolescentes }(n=1528) \\
\text { e adultos }(n=1163) \\
\text { brasileiros }\end{array}$ & $\begin{array}{c}\text { Para aferição de SAN utilizou-se a Escala } \\
\text { Brasileira de Insegurança Alimentar e } \\
\text { Nutricional (EBIA), além de medidas } \\
\text { antropométricas (aferidas). }\end{array}$ \\
\hline $\begin{array}{l}\text { Santos, } \\
\text { Gigante e } \\
\text { Domingues }^{42}\end{array}$ & 2010 & $\begin{array}{l}\text { Apresentar a prevalência } \\
\text { de IA em famílias, bem } \\
\text { como descrever o estado } \\
\text { nutricional dos membros } \\
\text { dessas famílias. } \\
\end{array}$ & $\begin{array}{l}1450 \text { domicílios } \\
\text { brasileiros }\end{array}$ & $\begin{array}{c}\text { Para aferição de SAN utilizou-se a Escala } \\
\text { Brasileira de Insegurança Alimentar e } \\
\text { Nutricional (EBIA), além de medidas } \\
\text { antropométricas (aferidas). }\end{array}$ \\
\hline $\begin{array}{l}\text { Velásquez- } \\
\text { Melendez et } \\
\text { al. }^{44}\end{array}$ & 2011 & $\begin{array}{c}\text { Determinar se a IA está } \\
\text { associada com o aumento } \\
\text { prevalência de obesidade } \\
\text { entre mulheres em idade } \\
\text { reprodutiva }\end{array}$ & $\begin{array}{l}\text { Mulheres brasileiras } \\
\text { em idade fértil } \\
\quad(n=10226)\end{array}$ & $\begin{array}{l}\text { Questionários sobre questões } \\
\text { socioeconômicas, estilo de vida, para } \\
\text { a medida de SAN utilizou-se a EBIA, e } \\
\text { medidas antropométricas (aferidas). }\end{array}$ \\
\hline Pan et al. ${ }^{45}$ & 2012 & $\begin{array}{l}\text { Examinar a associação entre } \\
\text { a obesidade e a IA em } 12 \\
\text { estados americanos }\end{array}$ & $\begin{array}{l}\text { Adultos americanos } \\
\qquad(\mathrm{n}=75103)\end{array}$ & $\begin{array}{l}\text { Medidas antropométricas foram relatadas, } \\
\text { além disso foi questionado variáveis } \\
\text { socioeconômicas, e aplicado questionário } \\
\text { de SAN que foi Household Food Security } \\
\text { Survey desenvolvido pelo US Department of } \\
\text { Agriculture (USDA). }\end{array}$ \\
\hline
\end{tabular}




\section{Considerações Finais}

A partir dessa revisão foi possível concluir que a obesidade teve relação com a IA e nutricional. Além disso, o enfoque nos estudos apresentados era principalmente nas mulheres. Estudos com uma população tão específica foi uma limitação do presente trabalho. Houve diferentes medidas utilizadas tanto para a exposição e o desfecho nos estudos; algumas análises restringiram média e baixa renda familiar, o que seria importante para avaliar o impacto de tais resultados.

Sugere-se 0 aperfeiçoamento da estrutura conceitual de que o IMC não seria o parâmetro ideal para avaliação da obesidade; a realização de estudos longitudinais, qualitativos e quantitativos, pode aumentar a compreensão da relação temporal entre IA e o ganho de peso e aumento do quadro de obesidade. Existe necessidade, ainda, de estudos em diversas populações, especialmente em adultos jovens e homens. Além disso, os estudos devem considerar as pessoas que recebem benefícios governamentais por períodos mais longos são propensos a ter características diferentes do que os indivíduos que recebem benefícios por períodos mais curtos, ou não recebem.

Sugere-se o desenvolvimento de consenso a respeito de uma ferramenta de avaliação padrão para determinar a IA, o que pode permitir melhores comparações entre os estudos nacionais e internacionais.

$\mathrm{O}$ aumento da disponibilidade de alimentos saudáveis, medidas regulatórias como a publicidade de alimentos e a melhora do perfil nutricional com educação alimentar e geração de conhecimento são alguns pontos chave para a busca da SAN e a diminuição da obesidade. A ampliação de políticas públicas que atrelem esses dois paradoxos é fundamental para a efetivação de melhoria nos quadros apresentados.

\section{Referências Bibliográficas}

1. Pinstrup-Andersen P. Food security: definition and measurement. Food Security. 2009 1(1):5-7.

2. Misselhorn A, Aggarwal P, Ericksen P, Gregory P, Horn-Phathanothai L, Ingram J, Wiebe K. A vision for attaining food security. Curr Opin Environ Sustainability. 2012 4(1):7-17.

3. Nordim SM, Boyle M, Kemmer TM . Position of the Academy of Nutrition and Dietetics: Nutrition Security in Developing Nations: Sustainable Food, Water, and Health. J Acad Nutr Diet. 2013 113(4):581-595.

4. Busse SR. Anorexia, Bulimia e Obesidade. Barueri, SP: Manole; 2004.

5. Ruhm CJ. Understanding overeating and obesity. J Health Econ 2012 31(1):781-796.

6. Cai L, He J, Song Y, Zhao K, Cui W. Association of obesity with socio-economic factors and obesity-related chronic diseases in rural southwest China. Public Health 2013 127(3):247-51.

7. Shils M, Shike M, Ross AC, Caballero S, Cousins RJ. Nutrição Moderna na saúde e na doença. $10^{a}$ edição. Barueri, SP: Manole; 2009.

8. Metallinos-Katsaras, E. et al. A Longitudinal Study of Food Insecurity on Obesity in Preschool Children. J Acad Nutr Diet 2012 Dec;112(12):1949-58

9. Dietz, W.H. Does hunger cause obesity? Pediatrics 1995 95(5):766.

10. Câmara Interministerial de Segurança Alimentar e Nutricional. Plano Nacional de Segurança Alimentar e Nutricional: 2012/2015. Brasília, DF: CAISAN; 2011.

11. Brasil. Princípios e Diretrizes de uma Política de Segurança Alimentar e Nutricional. Brasília; 2004.

12. Conselho Nacional de Segurança Alimentar e Nutricional - CONSEA. Princípios e Diretrizes de uma Política de Segurança Alimentar e Nutricional. Brasília, DF; 2004.

13. Valente FLS. Direito humano à alimentação: desafios e conquistas. São Paulo: Cortez; 2002.

14. Sage $C$. The interconnected challenges for food security from a food regimes perspective: Energy, climate and malconsumption. J Rural Studies 2013 29(1): 71-80.

15. Maluf RS, Menezes F, Marques SB. Caderno segurança alimentar [S.I.], 2000. Parte 12 - 13. Disponível em: <http://ag20. cnptia.embrapa.br/Repositorio/seguranca+alimentar_000gvxlxe0q02wx7ha0g934vgwlj72d2.pdf>. Acesso em 4 abr. 2013.

16. Rose DD. Interventions to reduce household food insecurity: a synthesis of current concepts and approaches for Latin America. Rev Nutr 2008 21:Suplemento:159s-73s.

17. Pinheiro ARO. A alimentação saudável e a promoção da saúde no contexto da segurança alimentar e nutricional. Rev CEBES: Saúde Debate 2005 29(70):125 -39. 
18. Franklin B, Jones A, Love D, Puckett S, Macklin J, White-Means S. Exploring Mediators of Food Insecurity and Obesity: A Review of Recent Literature. J Community Health. 2012 Feb;37(1):253-64.

19. Roth J, Qiang X, Marbán SL, Redelt H, Lowell BC. The obesity pandemic: Where have we been and where are we going? Obes Res. 2004 Nov;12 Suppl 2:88S-101S.

20. World Health Organization. Overweight and obesity. Genebra: WHO; 2011. (Fact sheet no. 311).

21. Kac G, Sichieri R, Gigante DP. Epidemiologia Nutricional. Rio de Janeiro: Editora Fiocruz/Atheneu; 2007.

22. Offer A, Pechey R, Ulijaszek S. Obesity under affluence varies by welfare regimes: The effect of fast food, insecurity, and inequality. Econ Hum Biol. 2010 Dec;8(3):297-308.

23. Campos P, Saguy A, Ernsberger P, Oliver E, Gaesser G. The epidemiology of overweight and obesity: public health crisis or moral panic? Int J Epidemiol. 2006 Feb;35(1):55-60.

24. Machado JDC, Silvestre SCM, Marchini, JS. Nutrição e Metabolismo: Manual de Procedimentos em Nutrologia. Rio de Janeiro: Guanabara Koogan; 2009.

25. Cuppari L. Nutrição Clínica no Adulto. $2^{a}$ edição. Barueri, SP: Manole; 2007.

26. Instituto Brasileiro de Geografia e Estatística - IBGE. Pesquisa de Orçamentos Familiares 2008-2009: Despesas, Rendimentos e Condições de Vida. Ministério do Planejamento, Orçamento e Gestão. Rio de Janeiro; 2010.

27. Oliveira ML. Estimativa dos custos da obesidade para o SUS. Tese de Doutorado. Programa de Pós-Graduação em Nutrição Humana da Faculdade de Ciências da Saúde da Universidade de Brasília; Brasília; 2013.

28. Dinour LM, Bergen D, Yeh MC. The Food Insecurity-Obesity Paradox: A Review of the Literature and the Role Food Stamps May Play. Am Diet Assoc 2007 107(11):1952-61.

29. Crawford PB, Webb KL. Unraveling the paradox of concurrent food insecurity and obesity. Am J Prev Med 2011 40(2):274-5. 30. Rutten LF, Yaroch AL, Patrick H, Story M. Obesity Prevention and National Food Security: A Food Systems Approach. IRSN Pub Health 2012 2012:1-10.

31. Kaiser LL, Townsend MS, Melgar-Quiñonez HR, Fujii ML, Crawford PB. Choice of instrument influences relations between food insecurity and obesity in Latino women. Am J Clin Nutr. 2004 Nov;80(5):1372-8.

32. Adams EJ, Grummer Strawn L, Chavez G. Food Insecurity Is Associated with Increased Risk of Obesity in California Women. J Nutr 2003 133(4):1070-74.

33. JilcottSB, Wall-BassettED, Burke SC, Moore JB. Associations between Food Insecurity, Supplemental Nutrition Assistance Program (SNAP) Benefits, and Body Mass Index among Adult Females. J Am Diet Assoc. 2011 Nov;111(11):1741-5

34. Shariff ZM, Khor JL. Obesity and household food insecurity: evidence from a sample of rural households in Malaysia. Eur J Clin Nutr 2005 59:1049-58.

35. Wilde PE, Peterman JN. Individual Weight Change Is Associated with Household Food Security Status. J Nutr 2006 136(5). 36. Martin KS, Ferris AM. Food Insecurity and Gender are Risk Factors for Obesity. J Nutr Educ Beh 2007 39(1):31-6.

37. Hanson KL, Sobal J, Frongillo EA. Gender and Marital Status Clarify Associations between Food Insecurity and Body Weight. J Nutr 2007 137(6):1460-5.

38. Seligman HK, Bindman AB, Vittinghoff E, Kanaya AM, Kushel MB. Food Insecurity is Associated with Diabetes Mellitus: Results from the National Health Examination and Nutrition Examination Survey (NHANES) 1999-2002. J Gen Intern Med. $2007 \mathrm{Jul} ; 22(7): 1018-23$

39. Tayie FA, Zizza CA. Food insecurity and dyslipidemia among adults in the United States. Prev Med. 2009 May;48(5):480-5.

40. Olson CM, Strawderman MS. The Relationship Between Food Insecurity and Obesity in Rural Childbearing Women. J Rural Health. 2008 Winter;24(1):60-6.

41. Oliveira JS, Lira PIC, Veras ICL, Maia SR, Lemos MCC, Andrade SLLS et al. Estado nutricional e insegurança alimentar de adolescentes e adultos em duas localidades de baixo índice de desenvolvimento humano. Rev Nutr 2009 22(4):453-65.

42. Santos JV, Gigante DP, Domingues MR. Prevalência de insegurança alimentar em Pelotas, Rio Grande do Sul, Brasil, e estado nutricional de indivíduos que vivem nessa condição. Cad Saúde Púb 2010 26(1):41-9.

43. Borre K, Ertle L, Graff M. Working to Eat: Vulnerability, Food Insecurity, and Obesity Among Migrant and Seasonal Farmworker Families. Am J Ind Med. 2010 Apr;53(4):443-62.

44. Velásquez-Melendez G, Schlüssel MM, Brito AS, Silva AA, Lopes-Filho JD, Kac G. Mild but Not Light or Severe Food Insecurity Is Associated with Obesity among Brazilian Women. J Nutr. 2011 May;141(5):898-902

45. Pan L, Sherry B, Njai R, Blanck HM. Food Insecurity Is Associated with Obesity among US Adults in 12 States. J Acad Nutr Diet. 2012 Sep;112(9):1403-9. 
46. Ibase. Repercussões do Programa Bolsa Família na Segurança Alimentar e Nutricional das Famílias Beneficiadas: relatório Síntese. Rio de Janeiro: Ibase; 2008.

47. Panigassi G, Segall-Correa AM, Marin-Leon L, Perez-Escamilla R, Sampaio MFA, Maranha LK. Food insecurity as an indicator of inequity: analysis of a population survey. Cad Saúde Púb 2008 24:2376-84.

48. Olson CM. Nutrition and health outcomes associat-ed with food insecurity and hunger. J Nutr 1999129 (Suppl.2): 521S-24S. 49. Alaimo K, Olson CM, Frongillo EA. Low family income and food insufficiency in relation to over-weight in US children: is there a paradox? Arch Ped Adol Med [serial on the Internet] 2001 [cited 2006 Mar 28]; 155 [about7p.].Available from: http://archpedi.amaassn.org/cgi/search?fulltext=Alaimo\&quicksearch_submit.x=4\&quicksearch_submit.y=6.

50. Caballero B. Subnutrição e obesidade em países em desenvolvimento. In: Cadernos de Estudos. Desenvolvimento Social em Debate, n. 2. Brasília: Ministério do Desenvolvimento Social e Combate à Fome;, 2005:10-3.

51. Kepple AW, Segall-Corrêa AM. Conceituando e medindo segurança alimentar e nutricional. Ciênc Saúde Coletiva 2011 16(1):187-99.

52. Jones SJ, Frongillo EA. Food insecurity and subsequent weight gain in women. Public Health Nutr. 2007 Feb;10(2):145-51. 53. Nord M, Andrews M, Winicki J. Frequency and duration of food insecurity and hunger in US households. J Nutr Educ Behav. 2002 Jul-Aug;34(4):194-200.

54. Lyons AA, Park J, Nelson CH. Food Insecurity and Obesity: A Comparison of Self-Reported and Measured Height and Weight. Am J Public Health. 2008 Apr;98(4):751-7.

\section{Caryna Eurich Mazur}

Endereço para correspondência - Rua: Praça Santos Andrade, n 39 , Bairro: Centro, CEP:80020-300 Curitiba, PR, Brasil.

E-mail: carynanutricionista@gmail.com

Lattes: http://lattes.cnpq.br/6817895976879675

Francisco Navarro - navarro@uol.com.br

Enviado em 06 de novembro de 2013. Aceito em 16 de julho de 2015. 